\title{
LAS FUENTES DE INFORMACIÓN EN AGRICULTURA DE REGADÍO. ESTUDIO Y EVOLUCIÓN DE LA SUPERFICIE DE OLIVAR DE RIEGO EN ANDALUCÍA. COMPARACIÓN CON LA PROVINCIA DE JAÉN
}

\author{
Molina Moral, Juan Carlos (1) (P). Pérez Latorre, Francisco José. (2).
}

Campus Científico Tecnológico Linares, Universidad de Jaén. Cinturón Sur s/n 23700 Linares.

1 Investigador, Universidad de Jaén. E-mail: jcmm0011@red.ujaen.es

${ }^{2}$ Profesor, Área Mecánica de Fluidos , Universidad de Jaén. E-mail; fjperez@ujaen.es

\section{Resumen.}

Conocer y determinar con la mayor precisión posible la superficie de riego de los cultivos, es un punto de partida necesario para posteriores estudios de caracterización de las instalaciones desde la perspectiva de análisis hídrico y energético. Es necesario también tener en cuenta otros aspectos como el origen de las aguas utilizadas, superficie regada, sistema de riego empleado, desniveles existentes, número de estructuras de almacenamiento/regulación, características de las redes de riego, número y potencia de los sistemas de bombeo, etc. Disponer de esta información se hace más interesante ante la posible transformación del modo de cultivo con la adopción de los sistemas intensivos, o en la aplicabilidad de estrategias de riego deficitario en grandes regiones. La consulta de diferentes Organismos Públicos pone de relieve, de forma habitual, que éstos presenten criterios distintos y utilizan metodologías diferentes para determinar la superficie de riego, dificultando la comparación de la información obtenida, de modo que en la mayoría de las ocasiones los resultados no son coincidentes. A ésta situación se podría añadir la discrecionalidad con la que se publican dichos datos. Las distintas metodologías de recogida y tratamiento de los datos, así como las fuentes principales de los mismos, son abordadas en el presente trabajo.

\begin{abstract}
"Abstract".
Know and determine with the major possible precision the crops irrigation surface is a necessary starting point for further research studies of characterization of the facilities from the perspective of water and energetic analysis. It is also necessary to take into account other aspects such as the origin of the used waters, watered irrigation, system of irrigation, differences in levels, number of structures of storage/regulation, characteristics of irrigation networks, number and power of the systems of pumping systems,..., etc. Having this information becomes more interesting in view of the possible transformation of the cultivation mode with the adoption of intensive systems, or in the applicability of deficit irrigation strategies in large regions. The consultation of different Public Organizations shows, in a habitual way, that they present different criteria and use a different methodology to determine the surface of irrigation, making difficult the comparison of the obtained information, so that in most of the occasions the results they are not coincidental. To this situation could be added the discretion with which data are published. The different methodologies of data collection and treatment, as well as the main sources thereof, are exposed in the present work, with the objective of determining the irrigation surface of olive groves at Andalusia, and particulary at Jaén's province, for further studies from the optics of water and energy efficiency.
\end{abstract}




\section{1) Introducción}

En la actualidad, en España a consecuencia de un profundo cambio estructural y determinados condicionantes ocurridos desde los primeros años del siglo pasado han originado que hoy en día tan solo el $3 \%$ de su población figure como agricultora, y a pesar de ello es uno de los países que mayor porcentaje de su territorio utiliza para uso agrícola y el medio rural, representando el $90 \%$ del territorio y el $20 \%$ de la población, (Robledo $R$, 2011). Así en el siglo $X X$ se ha pasado de tener poco más de un millón de hectáreas transformadas en riego a las aproximadamente 3.488.000 has existentes en la actualidad. Esta cifra, que supone el $18,3 \%$ de la superficie total de cultivo y el $13 \%$ de la Superficie Agraria Útil (SAU), produciendo del $55 \%$ al $60 \%$ de la Producción Final Agraria (PFA), (Plan Nacional de Regadíos). Además, aproximadamente la mitad de la población activa agraria depende del regadío (Berbel J., 2007).

Hoy día, las estadísticas agrarias, aun siendo realizadas por el Ministerio, están integradas en los Planes Estadísticos Nacionales, (PEN), que tienen una vigencia de 4 años, y que se enmarcan dentro del PEN 2017-2020, y coordinadas con el INE (Instituto Nacional de Estadística) y su homólogo autonómico IEA, (Instituto de Estadística de Andalucía) cristalizando en la publicación de los Anuarios de Estadística Agraria, de carácter anual, desde inicios del siglo pasado, con algunos paréntesis.

A nivel autonómico, la Consejería de Agricultura, Pesca y Desarrollo Rural de la Junta de Andalucía, elaboró y publicó en tres ocasiones; 1997, 2002 y 2008 el ICRA, "Inventario y Caracterización de los Regadíos de Andalucía", que tuvo vigencia durante el período 1996-2008, empleando como fuente de datos además de las previamente citadas, la realización de encuestas y entrevistas directas a las entidades vinculadas con la agricultura de regadío y técnicas cartográficas. Además ha venido estableciendo la planificación y asignación de recursos al regadío, y concretamente al sector del olivar, (CAP,2011-20142019)

Por otra parte, la Directiva 2000/60/CE, de 23 de octubre del Parlamento Europeo y del Consejo, denominada como Directiva Marco del Agua, estableció la realización de Planes Hidrológicos de Cuenca por los organismos de cuenca, denominados como Confederaciones Hidrográficas, que se revisan y actualizan cada seis años, si bién antes de esta disposición europea ya se realizaban en España desde 1998, en virtud de la Ley de Aguas, (Ley 29/1985, de 2 de agosto). Hasta el momento han habido tres ciclos: 2009-2015, 2015-2021, (actualmente vigente), y el futuro 2021-2027, (CHG,2009-2015-2019). Esta actuación tuvo como punto de partida el informe final sobre la superficie de los cultivos de regadío y sus necesidades para el riego en la demarcación hidrográfica del Guadalquivir, (CHG, 2005).

Las cuencas y ríos que discurren por Andalucía se enmarcan en seis distritos hidrográficos distintos: tres intracomunitarios (Guadalete-Barbate, Odiel-Piedras, y Cuencas Mediterráneas Andaluzas; y tres intercomunitarios, (Guadalquivir, Segura y Guadiana), fijadas por el Real Decreto 125/2007, de 2 de febrero. En el caso particular de la provincia de Jaén, ésta queda afectada por dos cuencas, la del Guadalquivir y la del Segura. La cuenca hidrográfica del Guadalquivir en la provincia de Jaén ocupa casi la totalidad de la extensión de la misma con $12.892 \mathrm{~km}^{2}$, y la cuenca hidrográfica del Segura cuenta con 594 $\mathrm{km}^{2}$, aproximadamente.

Los organismos de cuenca registran los datos de superficies, tanto de solicitudes como de concesiones, ya sean públicas o privadas, diferenciando las otorgadas, denegadas o en proceso de resolución, en su ámbito territorial. 
Finalmente, con carácter discrecional y puntual encontramos algunos trabajos realizados por entidades $u$ organismos públicos o privados, y autores reconocidos que utilizan diferente metodología tanto en la adquisición como en el tratamiento de la información en atención a los objetivos que persiguen, y presentación de sus resultados. Un ejemplo de ello es el observatorio Económico de la provincia, entidad pública perteneciente al ámbito de la Diputación Provincial de Jaén, que abordó un estudio del olivar de riego durante el período 1985-1998, y posteriormente realizó un estudio-dictamen sobre la rentabilidad económica de las explotaciones de olivar en la provincia en 2011, (CES. 2011).

\section{2) Objetivos}

El objetivo fundamental del presente Trabajo es determinar la superficie de riego de olivar en Andalucía, y particularmente en la provincia de Jaén, para su posterior estudio desde la óptica de la eficiencia hídrica y energética.

\section{3) Materiales y Métodos}

Para la obtención de los datos de partida se han consultado los siguientes organismos y administraciones:

Anuarios de Estadística Agraria, (AEA).

Los anuarios de estadística agraria no recogen aspectos tales como el origen de las aguas o tipología de riego, pero a nivel general si vienen informando de modo anual, de la superficie de riego y secano a nivel provincial. Inicialmente la recogida de datos se realizaba en base a los aforos de campo, prospecciones a entidades representativas y/o encuestas directas. En la actualidad, los datos se recogen de la información administrativa derivada de las declaraciones de los solicitantes de ayudas por superficies, así como el SIGPAC, (Sistema de Información Geográfica de Parcelas Agrícolas). Para ello, tiene en consideración como parcelas de riego como aquellas que son declaradas por los propios agricultores. Los datos son recogidos por el Ministerio de Agricultura (MAGRAMA), y las Delegaciones Provinciales de Agricultura de cada provincia.

A partir de 1999 aplican un cambio de la metodología volviendo a sus inicios y aportando datos sobre el número de árboles diseminados, además de otros relativos al rendimiento.

\section{Organismos de cuenca, (CHG, CHS).}

Los organismos de cuenca básicamente realizan el recuento de solicitudes, concesiones y actas de notoriedad, refiriendo sus datos a cuencas y distritos hidrográficos, utilizando criterios que distinguen entre olivar tradicional e intensivo, pero no permiten discernir a nivel municipal o provincial la información ofrecida ni tampoco la superficie de riego de las parcelas que estén pendientes de los trámites de concesión, o bien que tengan concedidos riegos deficitarios o riegos de emergencia.

Para la determinación de que una superficie se encuentre o no en regadío establecen tres procedimientos; en primer lugar la declaración/solicitud de su propietario o titular; en segundo lugar porque se hayan detectado infraestructuras para el riego y/o cultivos o zonas regadas, (utilizando para ello la cartografía existente empleando técnicas de fotointerpretación y teledetección; y finalmente, en tercer lugar, por la inspección que se realice para verificar la situación declarada o detectada, lo cual pone de relieve cierto grado de incertidumbre y de la complejidad de la situación. 


\section{Instituto Nacional de Estadística (INE) e Instituto de Estadística de Andalucía (IEA).}

Aporta datos a nivel provincial, comarcal y por cuencas hidrográficas, diferenciando el origen del agua de riego utilizada. EI INE toma como fuente de datos los suministrados por el Ministerio de Agricultura, principalmente los Censos Agrarios, (el último se realizó en 2009), y las encuestas sobre la estructura de las explotaciones agrícolas, (de carácter anual). EI IEA realiza las mismas labores que el INE a nivel autonómico, siguiendo las directrices de los Planes Estadísticos Nacionales.

\section{Inventario y Caracterización de los Regadíos de Andalucía, (ICRA).}

EI ICRA realizado por la administración autonómica, (Consejería de Agricultura y Pesca de la Junta de Andalucía), supuso un esfuerzo de la administración andaluza por realizar un sistema información territorial del regadío aproximado a la realidad que sirviera de base a su planificación. Se realizaron tres publicaciones; 1997, 2002, y 2008. Posteriormente publicó el informe "Inventario de Regadíos 2008 y su evolución en la última década", (CAP, 2011).

En el primer inventario del ICRA (1997) la unidad mínima de información era la Zona de Riego, (Z.R.), cuya delimitación se basaba en la información disponible (recintos SIGPAC de riego) y su intersección con los límites municipales e hidrológicos tras un trabajo de campo consistente en la elaboración de cuestionarios específicos para los diversos grupos de usuarios: Comunidades de Regantes, regantes singulares, técnicos, funcionarios de diversas Administraciones, Ayuntamientos y Cámaras Agrarias (hoy desaparecidas), y la posterior cartografía de las zonas de riego, completándolas y contrastándolas con diversas fuentes estadísticas. De esta manera se creó una cobertura denominada "Sistemas de Explotación de Recursos Hídricos", (SER), que no coincidía ni con la delimitada en el Plan Hidrológico de Cuenca, ni con la del organismo de cuenca, (CHG), por lo que hubo que contrastarla y ajustarla. La posible razón de este hecho es que los recintos SIGPAC definidos como regables podían presentar tres orígenes distintos; en primer lugar las propias declaraciones de los agricultores al solicitar éstos las ayudas comunitarias con carácter anual; en segundo lugar por la identificación y fotointerpretación de infraestructuras de riego en las parcelas; y finalmente, en tercer lugar, por las parcelas declaradas como de riego en base al catastro de rústica. En el primer caso, como en el olivar las ayudas no están vinculadas al secano o regadío, la fiabilidad de los datos aportados por los agricultores depende de su propia actitud.

En su última revisión de 2008, se definieron las Unidades de Agregación de Recintos, (U.A.), y las Zonas Homogéneas de Riego, (Z.H.R,). Las primeras estarían constituidas por Comunidades de Regantes o regantes individuales mayores de 200 has, y las segundas serían las restantes, teniendo en cuenta criterios de proximidad geográfica y homogeneidad de los regadíos, desglosando los datos por comarcas agrícolas.

\section{Observatorio económico de la provincia de Jaén, (CES).}

La primera publicación relativa al olivar la realizó este organismo en 1998 (Consejo Económico y Social de la Provincia de Jaén, 1997), en base a los datos de los Anuarios de Estadística Agraria en los períodos 1985-86 y 1997-98. Posteriormente, en 2011, realizó una segunda publicación denominada "Dictamen sobre el Análisis de la rentabilidad económica de las explotaciones de olivar en la provincia de Jaén", usando la misma base de información y determinaciones propias, elaborando un desglose por comarcas agrícolas y distintas tipologías o categorías agronómicas de olivar, en base a la publicación "El Olivar Andaluz", (CAP, 2002). No existe otra publicación análoga en las restantes provincias de Andalucía. 


\section{4) Resultados y Discusión}

\section{Anuarios de Estadística Agraria, (AEA).}

Los Anuarios de Estadística Agraria son la fuente de información más completa proporcionándonos datos, generalmente, con periodicidad anual. En la figura 1 se ha representado la evolución de la superficie de riego de olivar en Andalucía y en la provincia de Jaén durante el periodo de tiempo 1928-2019. Al analizarla se observa que a grandes rasgos la superficie total de olivar en Andalucía ha ido expandiéndose paulatinamente, así como el aumento de la superficie de riego en este cultivo encontrándose respecto de ésta última tres momentos clave; la década de los 80 , la primera década del segundo milenio, y finalmente a partir del año 2014, aunque en la actualidad la tendencia es a disminuir ligeramente.

Respecto de la provincia de Jaén, la primera expansión del olivar de regadío en la provincia tuvo lugar durante el período 1972-1998, mostrando una tendencia ascendente moderada, sin embargo, es durante la década correspondiente al período 1995-2005 cuando la expansión de la superficie de riego muestra una tendencia más acusada. Finalmente, la subida desde 2012 no se debe a una expansión del cultivo, sino que es consecuencia un cambio en la metodología por parte de la Consejería de Agricultura de la Junta de Andalucía al incorporar los datos del ICRA, los recintos SIGPAC y modificar su metodología.

Figura 1: Evolución de la superficie de riego de olivar.

Evolución de la superficie de Olivar en Andalucía

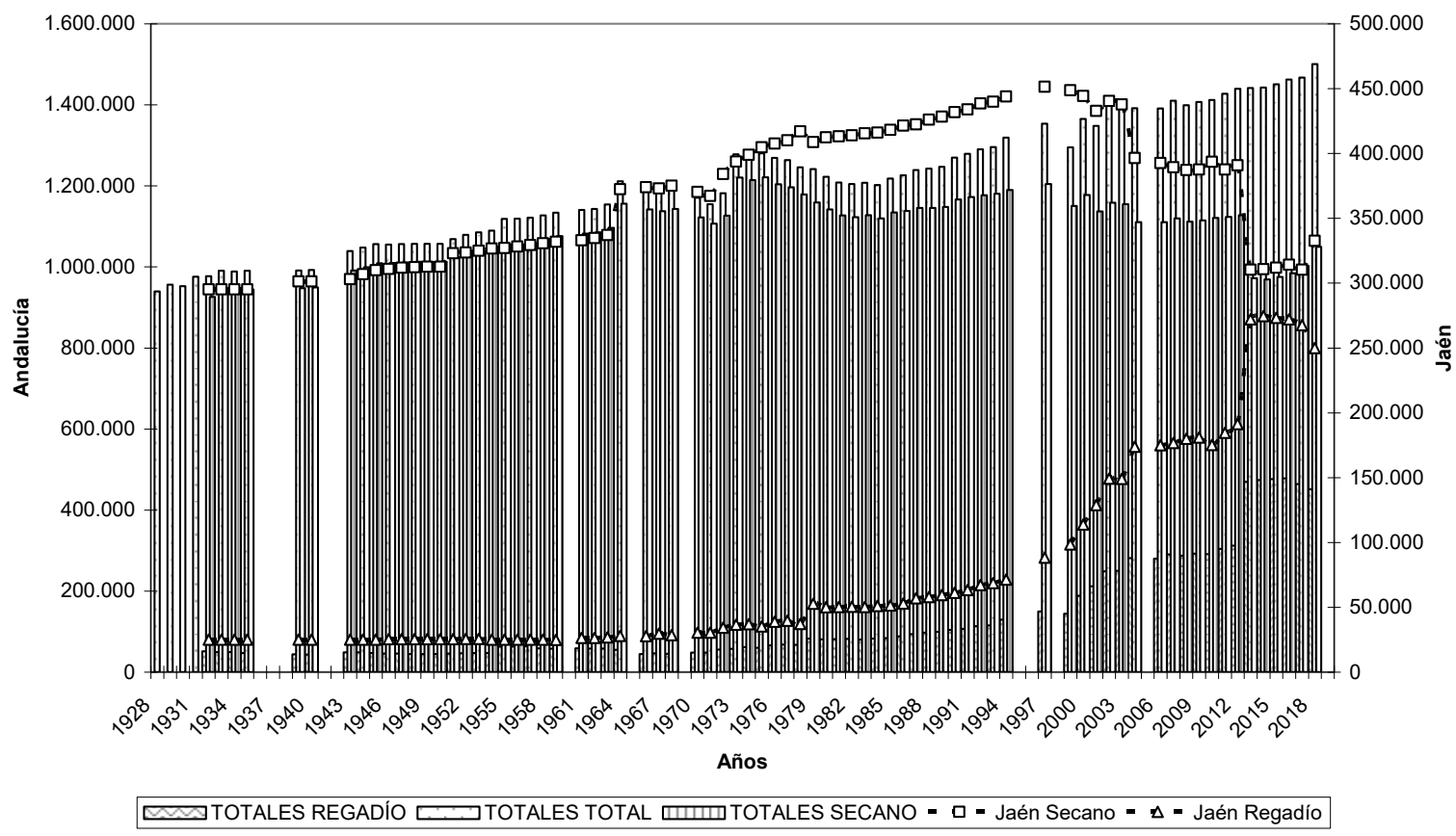

Fuente: AEA, Anuarios de Estadística Agraria y Delegación Provincial de Jaén, (1904-2019).

En un análisis de mayor detalle se ha tabulado la evolución del olivar en períodos quinquenales durante los últimos cuarenta años, diferenciando los datos de superficie total, secano y regadío (tablas 1 y 2 ). De este modo se pueden destacar incrementos muy acusados en el periodo 1995-2000 (de más del 41\%) y en el 2009-2012 (de más del 36\%). 
El continuo crecimiento se ha visto favorecido por el establecimiento de ayudas a la producción, y la ampliación favorable del mercado del aceite de oliva, así como ayudas específicas a la mejora de regadíos. También podemos destacar la relación entre olivar total y el de regadío, manteniéndose en valores próximos al $10 \%$ en el principio de la década de los años 90 del siglo pasado, y actualmente superando el $40 \%$.

Tabla 1:. Superficies de secano y regadío a nivel autonómico, por periodos de cinco años.

\begin{tabular}{|c|c|c|c|c|}
\hline \multicolumn{5}{|c|}{ Superficies medias de olivar en Andalucía. } \\
\hline Periodo & Secano, (has) & $\begin{array}{c}\text { Regadío } \\
\text { (has) }\end{array}$ & $\begin{array}{l}\text { Total } \\
\text { (has) } \\
\end{array}$ & $\begin{array}{c}\text { Sup. Regadío/Total } \\
\% \\
\end{array}$ \\
\hline $1976-1980$ & 1.175 .912 & 72.627 & 1.248 .539 & $5,82 \%$ \\
\hline 1981- 1985 & 1.126 .196 & 81.937 & 1.208 .133 & $6,78 \%$ \\
\hline 1986- 1990 & 1.148 .618 & 96.361 & 1.244 .978 & $7,74 \%$ \\
\hline 1991-1995 & 1.179 .799 & 115.929 & 1.295 .728 & $8,95 \%$ \\
\hline $1996-2000$ & 1.177 .311 & 160.466 & 1.337 .777 & $11,99 \%$ \\
\hline $2001-2005$ & 1.140 .051 & 247.666 & 1.387 .717 & $17,85 \%$ \\
\hline $2006-2010$ & 1.115 .484 & 288.157 & 1.403 .641 & $20,53 \%$ \\
\hline $2011-2015$ & 1.033 .373 & 406.911 & 1.440 .284 & $28,25 \%$ \\
\hline 2016-2019 & 1.012 .294 & 464.237 & 1.476 .531 & $31,44 \%$ \\
\hline
\end{tabular}

Fuente: AEA, Anuarios de Estadística Agraria, (1976-2019).

Tabla 2: Superficies de secano y regadío a nivel provincial, por periodos de cinco años.

\begin{tabular}{|c|c|c|c|c|}
\hline \multicolumn{5}{|c|}{ Superficies medias de olivar en la provincia de Jaén. } \\
\hline Periodo & Secano, (has) & $\begin{array}{c}\text { Regadío } \\
\text { (has) }\end{array}$ & $\begin{array}{l}\text { Total } \\
\text { (has) }\end{array}$ & $\begin{array}{c}\text { Sup. Regadío/Total } \\
\%\end{array}$ \\
\hline $1976-1980$ & 411.089 & 43.659 & 454.748 & $9,60 \%$ \\
\hline 1981- 1985 & 415.310 & 50.634 & 465.944 & $10,87 \%$ \\
\hline 1986- 1990 & 425.917 & 57.625 & 483.542 & $11,92 \%$ \\
\hline 1991-1995 & 439.012 & 67.540 & 506.552 & $13,33 \%$ \\
\hline $1996-2000$ & 448.092 & 100.178 & 548.271 & $18,27 \%$ \\
\hline 2001-2005 & 426.822 & 150.149 & 576.971 & $26,02 \%$ \\
\hline 2006-2010 & 390.051 & 177.560 & 567.612 & $31,28 \%$ \\
\hline 2011-2015 & 342.225 & 239.097 & 581.321 & $41,13 \%$ \\
\hline 2016-2019 & 318.973 & 263.109 & 582.082 & $45,20 \%$ \\
\hline
\end{tabular}

Fuente: AEA, Anuarios de Estadística Agraria, (1976-2019).

\section{Organismos de Cuenca, (CHG, CHS).}

Realizan escasas publicaciones, discrecionales, y desglosadas según parámetros hidrológicos, sin divisiones administrativas, con datos aislados y desagregados sin conexión ni continuidad temporal, (tabla 3). 
Tabla 3: Superficies de regadío a nivel autonómico según organismos de cuenca en Andalucía.

\begin{tabular}{l|cccccc} 
& CHG & CHCMA & CHGA & CHGB & CHS & CHTOP \\
\hline $\mathbf{1 9 9 9}$ & & & 20.936 & & & \\
$\mathbf{2 0 0 4}$ & 292.818 & & & & & \\
$\mathbf{2 0 0 5}$ & & & & & 17.718 & \\
$\mathbf{2 0 0 8}$ & & 25.346 & & & & \\
$\mathbf{2 0 0 9}$ & 409.808 & & 27.930 & & & 82 \\
$\mathbf{2 0 1 5}$ & 440.097 & & & 77 & & \\
$\mathbf{2 0 1 9}$ & & & & 77
\end{tabular}

Fuente: Elaboración propia a partir de Planes Hidrológicos de Cuenca vigentes.

En cuanto a la superficie de riego en las tablas 4 y 5 se pueden apreciar los datos correspondientes al año 2017, pudiéndose distinguir olivar tradicional y olivar intensivo y a la superficie regada cuando el olivar se asocia con otro cultivo.

Tabla 4: Superficie regada y regable de olivar en Jaén.

\begin{tabular}{cccc}
\hline & $\begin{array}{c}\text { SUPERFICIE } \\
\text { REGADA } \\
\text { (has) }\end{array}$ & $\begin{array}{c}\text { SUPERFICIE } \\
\text { REGADA OLIVAR Y } \\
\text { OTROS MÁS } \\
\text { (has) }\end{array}$ & $\begin{array}{c}\text { SUPERFICIE } \\
\text { REGABLE } \\
\text { (has) }\end{array}$ \\
\hline Olivar & $231.611,64$ & $239.352,79$ & $240.027,94$ \\
Olivar intensivo & $31.870,16$ & $46.413,46$ & $48.970,83$ \\
\hline TOTAL & $\mathbf{2 6 3 . 4 8 1 , 8 0}$ & $\mathbf{2 8 5 . 7 6 6 , 2 5}$ & $\mathbf{2 8 8 . 9 9 8 , 7 7}$
\end{tabular}

Fuente: CHG, Organismo de cuenca, 2018. . (Datos de 2017).

Tabla 5: Tipología de olivar en función del origen del agua en Jaén.

Olivar tradicional

\begin{tabular}{|c|c|c|c|}
\hline $\begin{array}{c}\mathrm{N}^{\circ} \mathrm{de} \\
\text { concesiones }\end{array}$ & Origen del Agua & $\begin{array}{l}\text { Sup. Regada } \\
\text { (has) }\end{array}$ & $\begin{array}{l}\text { Sup. Regable } \\
\text { (has) }\end{array}$ \\
\hline 178 & AGUAS SUBTERRÁNEAS & $121.664,95$ & $121.967,29$ \\
\hline 188 & AGUAS SUPERFICIALES & $103.501,41$ & $103.794,03$ \\
\hline 7 & \multirow{2}{*}{$\begin{array}{c}\text { ESCORRENTÍA } \\
\text { AGUAS REUTILIZADAS } \\
\text { (EDAR) }\end{array}$} & 987,61 & 987,61 \\
\hline 21 & & $13.198,82$ & $13.279,01$ \\
\hline 394 & & $239.352,79$ & $240.027,94$ \\
\hline \multicolumn{4}{|c|}{ Olivar intensivo } \\
\hline $\begin{array}{c}\mathbf{N}^{\circ} \text { de } \\
\text { concesiones }\end{array}$ & Origen del Agua & $\begin{array}{l}\text { Sup. Regada } \\
\text { (has) }\end{array}$ & $\begin{array}{l}\text { Sup. Regable } \\
\text { (has) }\end{array}$ \\
\hline 6 & AGUAS SUBTERRÁNEAS & $3.886,27$ & $3.886,27$ \\
\hline 49 & \multirow{3}{*}{$\begin{array}{c}\text { AGUAS SUPERFICIALES } \\
\text { ESCORRENTÍA } \\
\text { AGUAS REUTILIZADAS } \\
\text { (EDAR) }\end{array}$} & $42.527,19$ & $45.084,56$ \\
\hline 0 & & 0 & 0,00 \\
\hline 0 & & 0 & 0,00 \\
\hline 55 & & $46.413,46$ & $48.970,83$ \\
\hline 449 & & $285.766,25$ & $288.998,77$ \\
\hline
\end{tabular}


Fuente: CHG, organismo de cuenca, 2018. (Datos de 2017).

Los datos ofrecidos ponen de relieve que la variación final entre la superficie regada y regable es de 25.516,97 has. En cambio, si se considera la la superficie de olivar con otros cultivos asociados la diferencia se reduce a $3.232,52$ has. Si distinguimos entre superficie regable y regada de olivar con otros cultivos tenemos una diferencia de 675,15 has para el olivar tradicional y $2.557,37$ has en olivar intensivo.

Respecto de las concesiones otorgadas observamos que el olivar tradicional supone el $46,74 \%$ y el olivar intensivo el $53,26 \%$ respecto del total.

En relación al origen del agua tenemos que el empleo de aguas de escorrentía y residuales solo se utiliza en olivar tradicional, en el que por otra parte la utilización de aguas subterráneas y superficiales también se encuentra próxima al $50 \%$. En cambio el olivar intensivo presenta una mayor utilización de aguas superficiales, con el $92,06 \%$, frente a las aguas subterráneas, que arrojan un valor de $7,94 \%$ respecto del total.

Con respecto a la tipología de riego este es mayoritariamente localizado en el momento actual con el $90,92 \%$ de la superficie regada, seguido muy de lejos por el riego de superficie con un $7,17 \%$, y finalmente con un residual uso del riego por aspersión con un $1,91 \%$.

\section{Otras fuentes de información}

Bajo este epígrafe englobamos los datos ofrecidos por el INE, IEA, ICRA y CES, que básicamente adquieren los mismos de las dos principales fuentes de información previamente analizadas. Se ha de señalar que, los datos recogidos en el ICRA se adquieren con otra metodología e incluyen a las parcelas con riegos deficitarios y/o riegos de emergencia. También se han considerado los datos arrojados por otros autores de reconocido prestigio que recogen los datos suministrados por las fuentes oficiales, realizan estimaciones, o utilizan como base la cartografía para inferir la superficie de olivar de riego.

\section{Síntesis de resultados}

Al compararse los datos de las distintas fuentes de información disponibles, así como los referidos a trabajos publicados de diferentes autores que reflejan con carácter histórico las diferencias entre los mismos se observa que hasta el año 1995 no empiezan a aparecer diferencias significativas, que se mantienen fluctuando, hasta el año 2011, cuando comienzan a mostrar cierta convergencia. Así por ejemplo en el año 1991, según el organismo de cuenca (CHG), Jaén contaba con 62.000 has de riego de olivar, y en 1996 con 156.000 has (Saura, J., 1998). Este mismo año, el estudio monográfico del olivar realizado por el Observatorio Económico de la Provincia de Jaén expresaba según datos facilitados por la Delegación Provincial de Jaén, de la Consejería de Agricultura y Pesca de la Junta de Andalucía, que la superficie de olivar de regadío en la provincia era de 111.726 has, (CES, 1997).

De otro lado, la Consejería de Agricultura y Pesca de la Junta de Andalucía, en la elaboración del "Inventario y Caracterización de los Riegos de Andalucía", ofrece en 1997 valores intermedios respecto de los datos recabados por el Ministerio de Agricultura y el organismo de cuenca, pero a partir de 1998 arroja valores superiores a los ofrecidos por el primero, (estando ausentes los datos del segundo al no realizar un desglose por límites administrativos). 
De otro lado, los datos ofrecidos por distintos autores reconocidos arrojan valores ligeramente superiores a los de los Anuarios de Estadística Agraria.

Para poner de relieve los resultados obtenidos, se han elaborado las tablas 6.a, y 6.b, en las que se aborda parte de la información en períodos quinquenales recopilada considerando los casos en los que tenemos distintos valores de datos y aplicando técnicas de estadística descriptiva, debido a que las variables presentan observaciones limitadas.

Tabla 6.a: Tasa de variación. Andalucía.

\begin{tabular}{|c|c|c|c|c|c|c|}
\hline Período & AEA & CHG & ICRA & CAP & CES & $\begin{array}{c}\text { Otras } \\
\text { fuentes de } \\
\text { información }\end{array}$ \\
\hline 1976-1980 & 72.627 & & & & & \\
\hline 1981- 1985 & 81.937 & & & & & \\
\hline 1986- 1990 & 96.361 & & & & & \\
\hline 1991-1995 & $\begin{array}{l}115.929 \\
27,39 \%\end{array}$ & 91.000 & & & & $\begin{array}{l}1.094 .000 \\
1102,20 \%\end{array}$ \\
\hline $1996-2000$ & 160.466 & $\begin{array}{l}229.627 \\
43,10 \%\end{array}$ & $\begin{array}{l}231.961 \\
44,55 \%\end{array}$ & $\begin{array}{l}195.330 \\
21,73 \%\end{array}$ & & $\begin{array}{c}230.767 \\
43,81 \%\end{array}$ \\
\hline 2001-2005 & $\begin{array}{c}247.666 \\
7,59 \%\end{array}$ & $\begin{array}{l}277.905 \\
20,73 \%\end{array}$ & $\begin{array}{r}422.212 \\
83,42 \%\end{array}$ & & & 230.193 \\
\hline 2006-2010 & $\begin{array}{c}288.157 \\
7,46 \%\end{array}$ & $\begin{array}{c}409.808 \\
52,83 \%\end{array}$ & $\begin{array}{r}297.444 \\
10,92 \%\end{array}$ & 268.151 & & $\begin{array}{c}467.638 \\
74,39 \%\end{array}$ \\
\hline 2011-2015 & 406.911 & $\begin{array}{c}440.097 \\
8,16 \%\end{array}$ & & $\begin{array}{c}986.957 \\
142,55 \%\end{array}$ & & \\
\hline 2016-2019 & 464.237 & & & & & \\
\hline
\end{tabular}

Tabla 6.b: Tasa de variación. Jaén.

\begin{tabular}{|c|c|c|c|c|c|c|}
\hline Período & AEA & CHG & ICRA & CAP & CES & $\begin{array}{l}\text { Otras fuentes } \\
\text { de información }\end{array}$ \\
\hline $1976-1980$ & 43.659 & & & & & \\
\hline $1981-1985$ & 50.634 & & & & $\begin{array}{l}50.844 \\
0,41 \%\end{array}$ & $\begin{array}{l}50.844 \\
0,41 \%\end{array}$ \\
\hline 1986- 1990 & 57.625 & & & & & 56.029 \\
\hline 1991-1995 & $\begin{array}{r}67.540 \\
24,34 \%\end{array}$ & $\begin{array}{l}62.000 \\
14,14 \%\end{array}$ & & & $\begin{array}{c}74.577 \\
37,29 \%\end{array}$ & 54.321 \\
\hline $1996-2000$ & 100.178 & $\begin{array}{l}156.088 \\
55,81 \%\end{array}$ & $\begin{array}{l}157.800 \\
57,52 \%\end{array}$ & $\begin{array}{l}191.218 \\
90,88 \%\end{array}$ & $\begin{array}{c}105.081 \\
4,89 \%\end{array}$ & $\begin{array}{l}143.924 \\
43,67 \%\end{array}$ \\
\hline $\begin{array}{l}2001-2005 \\
2006-2010\end{array}$ & $\begin{array}{c}150.149 \\
620,17 \% \\
177.560\end{array}$ & 20.849 & $\begin{array}{c}202.237 \\
870,01 \% \\
189.023\end{array}$ & 261.140 & & $\begin{array}{c}156.057 \\
648,51 \% \\
181.510\end{array}$ \\
\hline
\end{tabular}




\begin{tabular}{c|c|c|c|c|c|c|} 
& & & $47,46 \%$ & $47,07 \%$ & $2,22 \%$ \\
$\mathbf{2 0 1 1 - 2 0 1 5}$ & 239.097 & & & 294.036 & 143.943 & 270.000 \\
& $66,11 \%$ & & & $104,27 \%$ & & $87,57 \%$ \\
$\mathbf{2 0 1 6 - 2 0 1 9}$ & 263.109 & 285.766 & & & &
\end{tabular}

A partir de los resultados, se puede afirmar que a nivel de Andalucía encontramos más datos durante los períodos del 1991-2015, y a su vez encontramos mayores divergencias.

La mayor tasa de variación tiene lugar durante el período 1991-1995, con el $1102,20 \%$, seguida del período $2006-2010$ con el 74,39\%. También podemos observar discrepancias significativas entre los datos ofrecidos por los AEA, (Anuarios de Estadística Agraria) y el organismo de cuenca, (CHG). que llegan a alcanzar valores máximos en torno al 3\% durante el período 1996-2000. También se pone de relieve que los datos ofrecidos por el ICRA, la Consejería de Agricultura y Pesca y distintos autores suelen ofrecer datos convergentes respecto al organismo de cuenca, pero arrojando discrepancias significativas.

A nivel de la provincia de Jaén obtenemos más datos en un amplio margen, desde 1981 a 2019 entre los datos ofrecidos. La mayor tasa de variación tiene lugar durante el período 2001-2005. Ilegando hasta el 90,88\%. y el período 2011-2015, que llega hasta el $140,25 \%$. Al comparar las distintas fuentes de información los valores del organismo de cuenca también muestran generalmente, con excepciones, valores superiores a los ofrecidos por los AEA. La Consejería de Agricultura y Pesca y el ICRA también ofrecen discrepancias durante los mismos períodos considerados; 1996-2000, y 2006-2010. Al igual que ocurre en el ámbito autonómico, los datos encontrados en otras fuentes de información correspondientes a autores de prestigio también ofrecen generalmente valores superiores al de los AEA, encontrando la máxima discrepancia durante el quinquenio 2001-2005.

El panorama general encontrado pone de manifiesto que existe una gran divergencia y dispersión de los datos.

\section{5) Conclusiones y Recomendaciones}

Se desprende del estudio realizado una falta de actualización de los datos recogidos pues en ocasiones la información existente se remite a fechas anteriores, los que nos lleva a determinar que no resultan válidos si queremos tomar decisiones acertadas. De igual forma se puede señalar que los organismos encargados de recabar información utilizan distintas metodologías, que además van modificando, y presentan una falta de uniformidad tanto en el tratamiento como en el desglose y publicación de los mismos, lo que arroja en numerosas ocasiones grandes divergencias que imposibilitan el conocimiento y la determinación con unos criterios básicos y generales de la superficie de riego del cultivo de olivar.

Se comprueba por orden de importancia que la principal fuente de información son los Anuarios de Estadística Agraria, y en segundo lugar la ofrecida por los Organismos de Cuenca, aunque arrojan discrepancias y diferencias significativas.

Se considera por tanto necesario la definición de una mejor y más clara estrategia de recogida de la información, con datos más actualizados, que posibilite determinar aspectos básicos como los usos, superficies, consumidores, precios, demandas, fuentes de agua, etc, siendo interesante así mismo el caracterizar los estudios a niveles más pequeños, por ejemplo, a nivel de municipio. En cualquier caso, con las precauciones precisas podemos afirmar que la superficie de olivar de riego en Andalucía se encuentra en torno a las 450.000 has, de las que más de la mitad se encuentran en la provincia de Jaén 250.000 has, lo que supone el $55,55 \%$, en 2018 . 
También se hace necesaria una mayor transparencia, periodicidad y publicación de la información recogida, el diseño de una estrategia de complementariedad de las metodologías empleadas, y finalmente la facilitación de su acceso, difusión y publicación libre de estos datos.

\section{6) Agradecimientos}

Queremos agradecer a D. Jesús González Delgado, Director Técnico del Departamento de Estudios y Estadísticas de la Delegación Provincial de Agricultura de Jaén su inestimable colaboración.

\section{7) Bibliografía}

Berbel, J., et al., (2007). "Multifuncionalidad del Regadío: Una aproximación empírica". Editorial: Universidad de Córdoba, (UCO). 15 p.

CAP, 2002. "El olivar andaluz". Empresa Pública de Desarrollo Agrario y Pesquero, (DAP. Unidad de Prospectiva. Difusión pública sin anexo. Consejería de Agricultura y Pesca, Junta de Andalucía .134 p.

CAP, 2008. "Inventario y Caracterización de los regadíos de Andalucía", Consejería de Agricultura y Pesca, Junta de Andalucía. CD, 13 p.

CAP, 2011. "Inventario de Regadíos 2008 y su evolución en la última década". Consejería de Agricultura y Pesca, Junta de Andalucía. 146 p.

CAP, 2011. Agenda del Regadío Andaluz. Horizonte 2015. Consejería de Agricultura y Pesca, Junta de Andalucía. 128 p.

CAP, 2014. "Diagnóstico de Situación Actual". Programa de Desarrollo Rural de Andalucía 2014-2020. Consejería de Agricultura y Pesca, Junta de Andalucía. 24-82.

CAP, 2014. "Programa de Desarrollo Rural de Andalucía 2014-2020. Subprograma temático del sector del Olivar". Consejería de Agricultura y Pesca, Junta de Andalucía. 59 p.

CAP, 2019. "Planificación Hidrológica 2021-2027". Consejería de Agricultura, Ganadería, Pesca y Desarrollo Sostenible, Junta de Andalucía.

CES, 1997. "Observatorio económico de la provincia de Jaén". Consejo Económico y Social de la Provincia de Jaén. 196 p.

CES, 2011. "Dictamen sobre el análisis de la rentabilidad económica de las explotaciones de olivar en la provincia de Jaén". Consejería de Agricultura y Pesca, Junta de Andalucía .58 p.

CHG, 1998. "Plan Hidrológico Nacional". Ministerio de Medioambiente.

CHG, 2005. Informe Final "Superficie de los cultivos de regadío y sus necesidades de riego en la demarcación hidrográfica del Guadalquivir". Consejería de Agricultura y Pesca, Junta de Andalucía. $127 \mathrm{p}$.

CHG, 2009. "Planificación Hidrológica 2009-2015". Ministerio de Medioambiente. CHG, 2015. "Planificación Hidrológica 2015-2021". Ministerio de Medioambiente. 
CHG, 2018. Datos suministrados por la delegación provincial de Jaén de la Confederación Hidrográfica del Guadalquivir.

CHG, 2019. "Tercer ciclo de Planificación 2021-2027". Memoria. Apartado 4.3.2.3.1. Ministerio para la Transición Ecológica. Confederación Hidrográfica del Guadalquivir. Págs: 183-188.

MAGRAMA, 1994-2008. "Plan Nacional de Regadíos". Fecha de aprobación: 1996. Horizonte: 2008.

MAGRAMA, 2006. " Plan de Choque de modernización de regadíos 2006-2008."

MAGRAMA, 2010. " Estrategia Nacional para la Modernización Sostenible de los Regadíos, horizonte 2015".

MAGRAMA, 2015. " Estrategia Nacional de Regadíos, horizonte 2020".

MAGRAMA, 2018. " Estrategia Nacional de Regadíos 2018-2025".

Robledo, R., 2011. "Historia del Ministerio de Agricultura 1900-2008. Política Agraria y Pesquera de España". Ed: MAGRAMA. 15p.

Saura, J., 1998. "La política del agua en el olivar. Los recursos hídricos del olivar en Andalucía". Foro del Olivar y del Medioambiente. Expoliva. 8 p. 